\title{
Educación virtual y enseñanza remota de emergencia en el contexto de la educación superior técnico-profesional: posibilidades y barreras
}

\author{
Virtual Education and Emergency Remote Teaching in the Context of \\ Technical-Professional Higher Education: Possibilities and Barriers
}

\author{
Carlos Ruz-Fuenzalida ${ }^{1}$
}

\begin{abstract}
RESUMEN
Las nuevas tecnologías y los alcances de la revolución digital no solo han permeado la educación escolar con herramientas y medios innovadores para la mejora de la enseñanza y aprendizaje de los/as estudiantes, sino que también se han constituido en un recurso de trabajo cada vez más relevante en la educación superior. La educación superior técnico-profesional representa un porcentaje importante de la matrícula del segmento terciario y tiene características particulares y de contexto que la diferencian sustancialmente de la educación universitaria. Teniendo lo anterior en consideración y en el marco de la pandemia por COVID-19, este artículo se refiere a la necesidad que enfrenta la educación superior técnico-profesional de implementar urgentemente formas de trabajo a distancia, lo que, a su vez, requiere buscar nuevas estrategias, caminos y enfoques que tengan en cuenta el uso de las diferentes herramientas de virtualidad que existen hoy en día.
\end{abstract}

Palabras claves: educación virtual; educación superior técnico-profesional; educación remota de emergencia; tecnología educativa; aprendizaje a distancia; pandemia; COVID-19.

\begin{abstract}
New technologies and advances of the digital revolution have permeated not only school education with innovative tools and means to improve teaching and learning, they also have become a relevant resource of work in higher education. Professional technical higher education represents an important percentage of the enrollment of tertiary education and has characteristics and context that differentiates it from college education. Considering this and in the framework of COVID-19 pandemic, this article refers to the need faced by technicalprofessional higher education to urgently implement forms of distance work which, in turn, requires seeking new strategies, paths and approaches that take in account the use of different virtuality tools that exist today.
\end{abstract}

Keywords: Virtual education; higher technical-professional education; remote emergency education; educational technology; distance learning; pandemic; COVID-19.

\footnotetext{
${ }^{1}$ Coordinador de Formación Transversal, Instituto Profesional CIISA, Santiago, Chile; Licenciado en Educación, Universidad Central, Santiago, Chile; cruz@ ciisa.cl.
} 


\section{Introducción}

La educación superior técnico-profesional se ha posicionado, durante los últimos años, como un segmento cada vez más relevante dentro de la matrícula anual del sistema de educación superior en Chile. Es así como su matrícula ha presentado un crecimiento sustancialmente mayor que su contraparte universitaria (Ruiz-Tagle y Paredes, 2019).

En el año 2010, la matrícula de primer año del segmento técnico-profesional superior se elevó por encima de la universitaria y para 2018 pasó a representar el 54\% de la matrícula total de primer año (Ruiz-Tagle y Paredes, 2019). Esto rompe con la concepción dominante en el imaginario colectivo social, que reconoce como horizonte de futuro de las personas la prosecución de estudios en el mundo universitario.

Es en este marco donde la virtualidad, junto con las herramientas digitales de enseñanza y aprendizaje, se sitúa como una transformación en la forma de trabajo con los/as estudiantes. Esta transformación ocurre no solo como producto de la revolución digital y las nuevas tecnologías, sino también por el entendimiento de que la sociedad de la información requiere que tanto la educación escolar como la educación superior sean capaces de enfrentar los retos que supone un cambio de época como el que vivimos actualmente.

La pandemia de COVID-19 que enfrenta la humanidad ha instalado un conjunto de estrategias de emergencia en la enseñanza de los/as estudiantes en general y, en particular, de aquellos/as que provienen del segmento superior técnico-profesional, los que, dado que poseen rasgos y características propias, demandan y exigen enfoques específicos para sus áreas y carreras.

A partir de lo anteriormente señalado, el presente artículo analiza la educación virtual en el segmento técnico-profesional superior en Chile, desde la perspectiva de los retos y desafíos que esta presenta, entendiendo que la pandemia que nos afecta (y seguirá afectando) implicará tomar decisiones que tendrán un gran impacto en la forma en la cual se trabaja con los/as estudiantes de este grupo tan importante de la educación terciaria en el país. 


\section{Características y evolución de la educación superior técnico-profesional en Chile}

La educación superior en Chile ha experimentado grandes transformaciones y cambios en los últimos 40 años. La serie de reformas al ecosistema de instituciones de educación superior, realizadas en 1981 bajo la dictadura cívico-militar, permitió la generación de nuevas instituciones, tanto universidades privadas como planteles técnico-profesionales. Desde entonces, se pasó de una matrícula de 118.978 estudiantes en 1980, a 1.268.510 en 2019 (Subsecretaría de Educación Superior [SIES], 2019), registrándose una tasa de incremento del 28,7\% solamente en el período 2010-2019.

En 2019 se declaraban 150 instituciones de educación superior, de las cuales 52 eran centros de formación técnica incluyendo 10 CFT estatales; además, existían 39 institutos profesionales, 19 universidades estatales del Consejo de Rectores de las Universidades Chilenas (CRUCH), 11 universidades privadas del CRUCH y 30 universidades privadas que no pertenecen al CRUCH. En cuanto al desglose de instituciones de educación superior con acreditación, en 2019 existían 15 centros de formación técnica acreditados, 19 institutos profesionales, 16 universidades estatales del CRUCH, 18 universidades privadas y 11 universidades privadas del $\mathrm{CRUCH}$, totalizando 79 instituciones de educación superior acreditadas (52,6\% del total) (SIES, 2020).

En este contexto, los diferentes tipos de instituciones que componen el sistema de educación superior conviven con significativas diferencias entre sí. Esto ha sido especialmente analizado por Ruiz-Tagle y Paredes (2019), tanto en su arquitectura jurídica como académica:

Mientras que las universidades pueden otorgan títulos técnicos, profesionales sin licenciatura y profesionales con licenciatura, las instituciones técnicas profesionales solo pueden entregar títulos técnicos y profesionales sin licenciatura. Del mismo modo, el otorgamiento de grados académicos solo está permitido para las instituciones universitarias. Ambos elementos limitan a los egresados de las instituciones técnicas profesionales la continuación de estudios y al acceso a ciertos trabajos en el sector público. (p.39) 
Otros aspectos que también marcan diferencias entre las instituciones de educación superior son la duración de los programas (entre dos a cuatro años), el precio anual de las carreras, el acceso al financiamiento estatal mediante becas y créditos, los requisitos de acceso (los institutos profesionales y los centros de formación técnica no forman parte del sistema único de acceso, como los planteles universitarios públicos y privados), así como también las variables asociadas a calidad a través de las acreditaciones (procesos a cargo del Consejo Nacional de Acreditación) (Ruiz-Tagle y Paredes, 2019).

Para el proceso de 2019, las universidades concentraron el 59,1\% de la matrícula total (considerando pregrado, postgrado y postítulo), mientras que los institutos profesionales se quedaron con el 30,1\% y los centros de formación técnica con el 10,9\%. Si consideramos el período de 2010 a 2019, son los institutos profesionales los que más crecen en comparación con los centros de formación técnica y las universidades, con una variación positiva del 70\% (SIES, 2019).

En relación a la matrícula total de pregrado para el año 2019, se mantuvo la concentración en las universidades con un 56,7\%, seguida por los institutos profesionales con un $31,8 \%$ y finalmente los centros de formación técnica con un 11,5\%. Lo relevante en este último caso es que, aun cuando fue leve el incremento de matrícula en comparación con el año anterior, las mayores alzas fueron en los institutos profesionales $(1,7 \%)$, seguidos por los centros de formación técnica $(0,9 \%) \mathrm{y}$, finalmente, las universidades (-0,2\%). Analizando el período completo de 2010-2019, las instituciones que más crecieron fueron los institutos profesionales $(69,2 \%)$, luego las universidades $(15,6 \%)$ y, finalmente, los centros de formación técnica (7,3\%) (SIES, 2019).

En materia de deserción en el sistema de educación superior, la evidencia indica que cerca del $30 \%$ de los/as estudiantes que se matriculan en primer año deserta de las instituciones de educación superior ${ }^{2}$. En este punto, los niveles mayores de deserción se encuentran en las instituciones técnico-profesionales, especialmente en carreras técnicas y en modalidad vespertina (Blanco et al., 2018).

\footnotetext{
${ }^{2}$ Las universidades estatales y privadas del CRUCH tienen tasas de deserción menores al 20\%. Por su parte, las universidades privadas que no pertenecen al CRUCH muestran una tasa en torno al $30 \%$, mientras que los institutos profesionales y los centros de formación técnica poseen tasas cercanas al 36\% y 37\%, respectivamente.
} 
Respecto a las principales características de quienes desertan del sistema, corresponden a hombres mayores de 25 años, pertenecientes a los quintiles de ingresos más bajos, provenientes de establecimientos escolares municipales (especialmente técnico-profesionales), con bajo rendimiento académico, bajo ranking de notas, bajos puntajes en pruebas como $\mathrm{SIMCE}^{3}$ o $\mathrm{PSU}^{4}$ y que carecen de ayudas o beneficios estudiantiles (Blanco et al., 2018).

\section{Elementos de la educación virtual en la educación superior}

La globalización y la irrupción de las nuevas tecnologías de la información, a través de la masificación de internet en las últimas décadas, han cambiado el panorama de las instituciones de educación superior en todo el mundo. La sociedad de la información y el conocimiento, como subproducto de los fenómenos descritos anteriormente, ha llevado al replanteamiento de los modos de enfrentar los nuevos desafíos que se presentan en todas las áreas del quehacer humano. Esto incluye, por supuesto, a la educación en general y, de manera específica, al ecosistema de la educación superior.

Es en este contexto donde surge la educación virtual como producto directo de las transformaciones sociales, políticas, económicas y culturales que han sido generadas como consecuencia de la globalización y las nuevas tecnologías de la información y comunicación (TIC). La educación virtual constituye un ecosistema educativo en donde se articulan elementos curriculares, pedagógicos, plataformas de trabajo y otras tecnologías, que permiten acceder, gestionar y construir aprendizajes significativos en los/as estudiantes (Herrera-Sánchez, 2016).

De esta forma, la educación virtual tiene como finalidad primordial la promoción de la formación, la investigación y el servicio, a través de las TIC, con diferentes intensidades y focos, según las necesidades propias de las personas.

En esta perspectiva, la educación virtual viene a complementar e integrar la función de la educación superior. Con ella es posible no solo la enseñanza, sino también la

\footnotetext{
${ }^{3}$ Sistema de Medición de la Calidad de la Educación.

${ }^{4}$ Prueba de Selección Universitaria.
} 
investigación, la extensión y la transferencia. Sin duda que el impacto de optimizar la gestión del aprendizaje es esencial en un mundo globalizado e interconectado, de ahí las ventajas que ofrece la educación virtual (Herrera-Sánchez, 2016).

Por otro lado, la entrada de la educación virtual en el segmento de la educación superior también ha creado nuevas condiciones de base para procesos de innovación educativa, los que se han visto favorecidos en estas últimas décadas. Muchos de ellos datan de al menos dos siglos atrás y ahora, con los recursos y medios disponibles, han podido desarrollarse y difundirse en el conjunto de las instituciones de educación superior (Chan, 2016).

Tanto en educación superior como a nivel escolar, la mediación que hacen las TIC en los procesos de enseñanza y aprendizaje se sustenta en modelos y enfoques que se conocen hace mucho tiempo y, por lo tanto, diversas iniciativas que hoy aparecen como vanguardistas e innovadoras en realidad no lo son tanto. Por ejemplo, la enseñanza personalizada surge en el siglo XVIII con Herbert (1776-1841) y más adelante, en el siglo XIX y la primera mitad del XX, es profundizada por Montessori (1870-1952) y Dewey (1859-1952) (Chan, 2016).

Hoy en día, cuando el flujo de datos e información en el mundo corren a una velocidad jamás vista, las ideas o proyectos en educación se convierten de forma muy sencilla en tendencias globales, muchas veces sin profundizar en su conceptualización y sustento teórico, de modo que no necesariamente llegarán a constituir estructuras de conocimiento con marcos epistemológicos adecuados.

Justamente el valor de la educación virtual es que abre la posibilidad de actualizar las estrategias y modelos educativos, cuyas opciones se amplían mediante las TIC. Dichas tecnologías hoy constituyen una tendencia que trasciende el ámbito educativo, siendo fuertemente impulsadas por intereses económicos, empresariales y culturales. Sin duda alguna, la virtualización, entendida como la capacidad de utilizar información y conocimiento proveniente del mundo digital para la toma de decisiones, es clave para la digitalización de la economía y la sociedad en su conjunto (Chan, 2016).

Claramente, la educación virtual representa mucho más que virtualizar campus y aulas, dado que apunta a un cambio en la gestión conjunta de los ambientes y entornos de 
aprendizaje, tanto físicos como digitales, en donde las TIC constituyen verdaderos puentes para la representación de los diferentes objetos del conocimiento y el modelamiento de las interacciones para el aprendizaje (Chan, 2016).

\section{Enseñanza remota de emergencia como respuesta ante la pandemia de COVID-19}

Qué duda cabe que la pandemia de COVID-19 está causando grandes estragos en todo el mundo y complicaciones nunca antes vistas. De acuerdo a los datos publicados por la Organización Mundial de la Salud (OMS), en junio de 2020 existían 6.287.771 personas infectadas, mientras que 379.941 habían fallecido. De esos totales, el 46,9\% de los casos activos y el 43,5\% de los fallecimientos correspondían al continente americano. En el caso de Chile, en junio de 2020 existían 108.686 casos confirmados y 1.188 fallecidos/as a raíz de este virus (Organización Mundial de la Salud [OMS], 2020).

Transversalmente, en todos los países y sectores del quehacer humano, se han visto trastocadas actividades habituales y comunes, muchas de ellas suspendidas o sencillamente detenidas hasta nuevo aviso. La educación como actividad humana no es ajena a esta pandemia y, tanto a nivel preescolar como escolar y superior, se han implementado respuestas contingentes, muchas de ellas improvisadas, para seguir con los procesos de enseñanza y aprendizaje a través de computadoras, tablets y smartphones.

En particular, las instituciones de educación superior se han volcado a transformar sus clases y procesos de trabajo, entre profesores/as y estudiantes, al formato en línea. En varias instituciones los/as estudiantes están recibiendo las horas de clases a través de una serie de plataformas remotas, tales como Microsoft Teams, Zoom, Jitsi, entre otras. Sin el contacto presencial entre los actores del proceso de enseñanza y aprendizaje, estudiantes y profesores/as avanzan mientras la contingencia continúa en el mundo (Abreu, 2020).

Bajo estas condiciones, la enseñanza remota de emergencia se convirtió, con el paso de las semanas y desde el comienzo de la crisis mundial, en la única opción viable para responder a la exigencia de continuar con los procesos de enseñanza y aprendizaje en 
todo el mundo. Escuelas, colegios, universidades, institutos y centros de formación técnica cerraron rápidamente sus campus y recintos de trabajo, para pasar directamente a una instrucción en línea, transformando la educación a distancia no en una opción, sino en una urgencia.

Uno de los elementos que deben tenerse presente en el contexto de la pandemia es que la educación en línea, específicamente la educación virtual, requiere tiempo de preparación, diseño, equipos de trabajo y recursos. Es ahí donde comienzan a diferenciarse la educación virtual y la enseñanza remota de emergencia. Esta última tomó a instituciones, centros educativos y cuerpos docentes sin tiempo para prepararse y con limitados recursos. La situación de la pandemia exigía una respuesta rápida con el fin de satisfacer una enorme demanda por servicios educativos en el mundo (Abreu, 2020).

Considerando que tanto estudiantes como profesores/as de las diferentes instituciones educativas, ya sean escolares o de educación superior, carecen de formación y capacitación en educación en línea, la apuesta ha sido ofrecer cursos que no fueron preparados o diseñados para esta modalidad. En general, la construcción de un curso en línea demanda un equipo multidisciplinario de trabajo, que realiza una construcción orgánica y sistematizada del producto que más tarde será ofrecido a los/as estudiantes.

Hay investigaciones que señalan que el éxito de la educación virtual depende directamente de tres factores esenciales: interacción con la materia a tratar, con los docentes que imparten el contenido y entre los mismos estudiantes. El aprendizaje en línea de calidad se producirá cuando estas tres condiciones se articulen de manera coherente y armónica, convirtiéndose así en un aprendizaje multidimensional. Ello dista de lo que hoy en día ocurre en muchas instituciones de educación superior, donde la relación que se establece es unidireccional y los/as estudiantes, al asistir a clases en línea, deben limitarse a escuchar las conferencias remotas de los/as docentes (Abreu, 2020).

La diferencia entre la educación virtual y enseñanza remota de emergencia es básicamente la experiencia de aprendizaje que el/la estudiante vive en su proceso de enseñanza. La enseñanza remota de emergencia es una apuesta a un cambio temporal en la provisión de educación a un grupo de estudiantes, en función del contexto de emergencia que se está viviendo. Considera a su vez soluciones mediante TIC para la 
instrucción o educación que sean equivalentes a las que normalmente sucederían de forma presencial. El objetivo, por tanto, no es contar con un ecosistema de enseñanza y evaluación sólido, sino más bien entregar acceso temporal a la instrucción y a los procesos educativos, de manera rápida y sencilla, ante una emergencia como la actual (Hodges et al., 2020).

En la medida en que comprendemos de mejor forma la enseñanza remota de emergencia, se entiende la diferencia con la educación virtual. Las exigencias que plantean la enseñanza remota de emergencia a los/as docentes, en cuanto al control de la asignatura, su desarrollo, proceso de implementación y evaluación, demandan apoyos de los equipos de diseño instruccional, desarrollo y directivos, con el fin de disponer de forma rápida de habilidades para trabajar y enseñar en un entorno en línea. Es así como los equipos de apoyo educativo deben reinventarse en tiempos de pandemia y de crisis, para enfrentar de forma colectiva los desafíos que generan situaciones como las que estamos viviendo en el mundo (Hodges et al., 2020).

La transición rápida a una enseñanza remota de emergencia implica dejar de lado, en muchos casos, la calidad de los cursos y procesos impartidos por las instituciones o centros educativos. Ahí es donde se aprecia la distancia que la separa de la educación virtual, que requiere meses de planificación y diseño, alta inversión en infraestructura y equipos de trabajo, así como también una correcta arquitectura de procesos de implementación. La enseñanza remota de emergencia no es una solución de largo plazo, sino una aproximación temporal a una circunstancia inmediata y transitoria, que en este caso corresponde a la pandemia de COVID-19 (Hodges et al., 2020).

\section{Complejidades de la educación virtual y la enseñanza remota de emergencia en el segmento técnico-profesional superior}

La educación técnico-profesional, especialmente en el nivel terciario o superior, es fundamental para el desarrollo de habilidades y competencias asociadas a la empleabilidad, fundamentalmente en sectores más pobres y carentes de oportunidades de movilidad social. De ahí que este segmento educativo genere un efecto de impacto en la 
cualificación de la persona, la que no termina una vez que ha ingresado al mundo laboral (Arroyo y Pacheco, 2017).

Como se indicó al comienzo del artículo, la educación superior técnico-profesional ha presentado un enorme crecimiento en el último tiempo. Esto no solo se refiere a un aumento en la cobertura y acceso a institutos profesionales y centros de formación técnica, sino que además involucra nuevos instrumentos de financiamiento público y privado, los cuales han permitido que miles de estudiantes ingresen a planteles que, casi en su totalidad, son privados (SIES, 2019).

En lo específico, este segmento de la educación superior es estratégico para el país porque posee el potencial de orientar la educación a fines propiamente productivos, con carreras técnicas y profesionales cortas y con alto acceso. Este punto es relevante, ya que es la educación técnico-profesional superior la que debería ser relevada como prioridad nacional, dada su importancia para el desarrollo del país.

Los/as estudiantes que son parte de la educación técnico-profesional superior son más propensos/as a asistir a una jornada vespertina que diurna. Esto podría explicarse por el hecho de que deben trabajar para financiar sus estudios y sostener sus hogares y familias. Por una parte, mientras que en 2007 solo el $20 \%$ de los/as estudiantes estaba en jornada vespertina, una década después esa cifra pasó a constituir el $43 \%$ dentro del ecosistema de institutos profesionales y centros de formación técnica. En esta línea, los/as estudiantes de este segmento provienen esencialmente de sectores más pobres y vulnerables (64\% de la matrícula de institutos profesionales y centros de formación técnica), en contraste con el segmento universitario, donde la procedencia es de quintiles más ricos en su mayor parte (Arroyo y Pacheco, 2017).

Si consideramos el origen de los/as estudiantes en educación superior, tenemos que el 45\% de quienes son parte del ecosistema de institutos profesionales y centros de formación técnica proviene de la modalidad técnico-profesional de enseñanza media, siendo muy diferente a lo encontrado en las universidades, donde solo el 17\% cursó esta modalidad de estudios. Este aspecto nos señala que el origen general de los/as estudiantes de este segmento de educación superior corresponde a hogares con menores recursos, acceso a servicios y oportunidades (Arroyo y Pacheco, 2017). 
La educación virtual y la enseñanza remota de emergencia en la educación técnicoprofesional superior presentan, por esta razón, complejidades y dificultades mayores que en el segmento universitario, debido fundamentalmente a la falta de recursos materiales, como también familiares, que faciliten un trabajo en línea pertinente. En la Región Metropolitana, la comuna de Cerro Navia, por ejemplo, presenta un nivel de acceso a internet de solo un 28,8\%, en comparación con San Miguel que tiene un 97,7\%. Otras comunas como La Pintana (28,2\%), Lo Espejo $(41,1 \%)$ y La Granja $(44,4 \%)$ muestran lo difícil del trabajo de una educación en línea dirigido a una población preferentemente técnica profesional (Subsecretaría de Telecomunicaciones, 2019).

En suma, el trabajo que puede realizarse en línea demanda, por parte de el/la estudiante, una serie de condiciones materiales y de acceso que en la práctica son complejas de disponer, siendo esto producto de las desigualdades territoriales que existen en nuestro país. Ante esto, tanto los institutos profesionales como los centros de formación técnica han respondido entregando diferentes recursos, como dispositivos de banda ancha móvil, tablets y notebooks, a modo de elementos remediales de trabajo. Sin embargo, en lo que hace referencia a aspectos de vivienda, entorno y hacinamiento, la desventaja de la educación técnico-profesional superior es muy amplia en comparación con la universitaria (Gajardo, 2019).

Más allá de las acciones remediales que puedan generarse a partir de iniciativas particulares de las diferentes instituciones de educación superior, el conjunto de actores involucrados en el ecosistema de educación superior técnico-profesional, tales como autoridades ministeriales, sector privado y organizaciones sociales, deben ser capaces de generar respuestas orgánicas y sistémicas para responder de forma adecuada y coherente a una realidad que subyace a la educación superior. La generación de políticas públicas en esta materia es fundamental, no solo para una mayor calidad del subsistema, sino que además para responder de forma inclusiva y pertinente a un segmento de la población que es crucial para el desarrollo del país. 


\section{Comentarios finales}

La pandemia de COVID-19 ha transformado completamente a la humanidad. Qué duda cabe que, una vez que logremos contar con tratamientos para los/as pacientes más críticos/as, los gobiernos y autoridades tendrán que replantearse -junto a la ciudadanía y las organizaciones sociales- la forma de convivir bajo una realidad diferente.

Cada uno de los sectores y actividades de la sociedad se ha enfrentado a fuertes ajustes en su desarrollo, en algunos casos con una reducción parcial o amplia en sus labores, mientras que en otros, sencillamente, se ha dado una paralización total, sin una visualización clara de un regreso a un estado anterior a esta emergencia sanitaria. Rubros como comercio, servicios, construcción, agricultura, entre otros, han sufrido fuertes variaciones en la oferta y la demanda de sus productos.

La educación, por su parte, representa un servicio que se ha visto dramáticamente afectado en su prestación a millones de personas y familias en el mundo. Según datos, más del 95\% de los niños, niñas y adolescentes en América Latina y el Caribe se encuentran fuera de las escuelas, colegios y liceos, como producto del cierre de los establecimientos a causa de la pandemia (Unicef Chile, 2020).

En el caso de Chile, la pandemia de COVID-19 ha producido la suspensión de las clases por parte del Ministerio de Educación en todo el sistema de educación preescolar y escolar, afectando a más de 3 millones de niños, niñas y adolescentes. Esto continúa hasta hoy en día, sin la certeza de cuándo podrá retornarse a la presencialidad en los diferentes establecimientos escolares, jardines infantiles y salas cunas.

En cuanto al sistema de educación superior, las diferentes instituciones han enfrentado desde marzo una suspensión general de actividades presenciales, tanto administrativas como académicas, movilizando a poco más de 1,2 millones de estudiantes a una experiencia de enseñanza remota de emergeencia, con todas las dificultades y complejidades que implica un tránsito rápido e improvisado a una modalidad donde existe, en general, falta de experiencia y habilidades por parte de docentes, estudiantes y otros actores del sistema. 
Por parte de la Superintendencia de Educación Superior, el foco estuvo puesto en el resguardo de la seguridad de los actores y de la calidad de la prestación de servicios educativos (Superintendencia de Educación Superior, 2020).

De manera específica, para el subsistema de educación superior técnico-profesional, el desafío de la migración a una enseñanza remota de emergencia ha sido aún más complejo. Con una matrícula de estudiantes que proviene de sectores más vulnerables, con falta de servicios básicos, conectividad, hacinamiento en viviendas y entornos desfavorecidos, los desafíos de aprendizaje son mucho mayores que los enfrentados por la mayoría de los planteles universitarios, cuyos estudiantes, en general, provienen de deciles con mayores ingresos.

Tanto los institutos profesionales como los centros de formación técnica se han volcado a disponer rápidamente de plataformas virtuales, junto con aplicaciones de videoconferencias, con el fin de que docentes y estudiantes puedan avanzar en los procesos de enseñanza y aprendizaje. Este camino se construye cada día y las dificultades no solo se limitan a contar con dispositivos, sino además con competencias digitales y habilidades para una educación en línea. Los resultados hasta ahora son heterogéneos y diversos, y claramente será interesante analizarlos desde la experiencia de sus actores principales.

Es importante tener presente que, aun cuando en el momento que se escribió este artículo la mayoría de las instituciones de educación ssuperior había logrado avanzar en el primer semestre académico, es improbable que se regrese a la presencialidad durante los próximos meses. Esto demanda, por parte de los diferentes planteles superiores, una estrategia con foco en conocer plenamente la realidad que tanto profesores/as como estudiantes han experimentado hasta ahora con la enseñanza remota de emergencia, no solo en su dimensión de aprendizajes, sino además en la emocional, afectiva y social. La información de la que dispongamos sobre este proceso, tanto en resultados como percepciones, permitirá dimensionar el impacto de la enseñanza remota de emergencia en los institutos profesionales y centros de formación técnica.

Lo que podamos aprender con esta emergencia sanitaria, especialmente en el caso de las instituciones técnico-profesionales, será crucial para enfrentar los desafíos que implica 
trabajar con estudiantes que vienen mayoritariamente de la enseñanza media técnicoprofesional, de los segmentos más pobres y vulnerables, y que además pertenecen a un subsistema escolar poco estudiado en su tránsito hacia la educación superior. Hoy el motor del desarrollo del país pasa necesariamente por este segmento de la educación superior, en donde la educación virtual y enseñanza remota de emergencia demandan un claro entendimiento de las dinámicas de este grupo de la población, siendo esto último una oportunidad de implementar mejores políticas públicas en la materia.

\section{Referencias}

Abreu, J. (2020). Tiempos de coronavirus: la educación en línea como respuesta a la crisis. International Journal of Good Conscience, 15(1), 1-15. http://www.spentamexico.org/v15-n1/A1.15(1)1-15.pdf

Arroyo, C., y Pacheco, F. (2017). Los resultados de la educación técnica en Chile. Comisión Nacional de Productividad. https://www.comisiondeproductividad.cl/wpcontent/uploads/2018/04/Nota_Tecnica_3_Educacion.pdf

Blanco, C., Meneses, F., y Paredes, R. (2018). Más allá de la deserción: trayectorias académicas en la educación superior en Chile. Calidad en la Educación, (49), 137-187. http://dx.doi.org/10.31619/caledu.n49.579

Chan, M. (2016). La virtualización de la educación superior en América Latina: entre tendencias y paradigmas. Revista de Educación a Distancia, (48), 1-32. https://doi.org/10.6018/red/48/1

Gajardo, S. (2019). Región Metropolitana de Santiago, Encuesta CASEN 2017: Resultados de Vivienda y Entorno. Secretaría Regional Ministerial de Desarrollo Social Región Metropolitana, Ministerio de Desarrollo Social. http://www.desarrollosocialyfamilia.gob.cl/storage/docs/DOCUMENTO_VIVIE NDA_Y_ENTORNO_RMS_CASEN_2017.pdf 
Herrera-Sánchez, G. (2016). Paradigma de la educación virtual y los nuevos escenarios de aprendizaje. Educación Superior, (21), 75-90. http://revistavipi.uapa.edu.do/index.php/edusup/article/view/113

Hodges, C., Moore, S., Lockee, B., Trust, T., \& Bond, A. (27 de marzo de 2020). The Difference Between Emergency Remote Teaching and Online Learning. Educause Review. $\quad$ https://er.educause.edu/articles/2020/3/the-difference-betweenemergency-remote-teaching-and-online-learning

Mi Futuro. (s.f.). Instituciones de Educación Superior en Chile. Recuperado el 31 de diciembre de 2019 de https://www.mifuturo.cl/instituciones-de-educacionsuperior-en-chile/

Organización Mundial de la Salud. (3 de Junio de 2020). Reporte de Situación - 135. https://www.who.int/docs/default-source/coronaviruse/situationreports/20200603-covid-19-sitrep-135.pdf?sfvrsn=39972feb_2

Ruiz-Tagle, C., y Paredes, R. (2019). Educación superior técnico profesional: ¿una alternativa a la universitaria? El Trimestre Económico, 86(341), 31-63. https://doi.org/10.20430/ete.v86i341.621

Superintendencia de Educación Superior. (19 de marzo de 2020). Mineduc anuncia Plan de acción para instituciones de educación superior por COVID-19. https://www.sesuperior.cl/mineduc-anuncia-plan-de-accion-para-institucionesde-educacion-superior-por-covid-19/

Servicio de Información de Educación Superior. (junio de 2019). Informe matrícula 2019 en educación superior en Chile. Subsecretaría de Educación Superior. https://www.mifuturo.cl/wp-content/uploads/2020/04/Informe-Matricula2019_SIES.pdf

Subsecretaría de Telecomunicaciones (s.f.). El Mapa de la Conectividad de la RM. Recuperado el 10 de diciembre de 2019 de https://www.subtel.cl/mapadigital/

Unicef Chile. (23 de marzo de 2020). COVID-19: Más del 95 por ciento de niños, niñas y adolescentes está fuera de las escuelas en América Latina y el Caribe. 
https://www.unicef.org/chile/comunicados-prensa/covid-19-m\%C3\%A1s-del95-por-ciento-de-ni\%C3\%B1os-ni\%C3\%B1as-y-adolescentes-est\%C3\%A1fuera-de-las 